\title{
THE ARGUMENT OF AN EXTREMAL DILATATION
}

\author{
MARVIN ORTEL AND WAYNE SMITH \\ (Communicated by Irwin Kra)
}

\begin{abstract}
Suppose $\kappa$ is the complex dilatation of an extremal quasiconformal homeomorphism of the unit disk onto itself. Then, except in special cases, the width of the set of arguments of $\kappa$ must strictly exceed $\pi$.
\end{abstract}

1. Introduction. In this paper, $\mathbf{C}$ is the set of complex numbers, $D \equiv\{z \in$ $\mathbf{C}:|z|<1\}$ is the unit disk, $\mathscr{L}^{2}$ is Lebesgue two dimensional measure on $\mathbf{C}$, and $\mathscr{L}^{1}$ denotes Lebesgue one dimensional measure on the set of real numbers $\mathbf{R}$. For $0<\theta<\pi$, the open sector with vertex angle $2 \theta$ about the positive real axis is denoted

$$
S(\theta) \equiv\left\{r e^{i t}: 0<r<\infty,|t|<\theta\right\} .
$$

We set $\operatorname{sgn} z \equiv z /|z|$ for $z \in \mathbf{C}-\{0\}$, and $\operatorname{sgn} 0 \equiv 0$.

If $\kappa$ is bounded and $\mathscr{L}^{2}$ measurable on $D$, then $\|\kappa\|_{\infty}$ denotes the infimum of the set of numbers $K$ such that $|\kappa(z)|<K$ for $\mathscr{L}^{2}$ almost all $z \in D$ : i.e.

$$
\|\kappa\|_{\infty} \equiv \operatorname{essip}_{z \in D}|\kappa(z)| \text {. }
$$

B shall denote the set of all functions, $f$, which are holomorphic on $D$ and satisfy

$$
\|f\|_{1} \equiv \int_{D}|f(z)| d \mathscr{L}^{2}(z)<\infty .
$$

In addition, $\mathbf{B}_{1} \equiv\left\{f \in \mathbf{B}:\|f\|_{1}=1\right\}$ denotes the boundary of the unit ball in B. In the current literature the Banach space $\mathbf{B}$ is often referred to as one of the Bergman spaces.

We introduce a constant, pertaining to $\mathbf{B}$, which will appear in our main theorem.

Let $C(\mathbf{B})$ denote the infimum of the set of all $C \in(0, \infty]$ such that

$$
\int_{D}|f(z)| d \mathscr{L}^{2}(z) \leq C \int_{D}|\operatorname{Re} f(z)| d \mathscr{L}^{2}(z)
$$

whenever $f \in \mathbf{B}$ and $\operatorname{Im} f(0)=0$.

In [Har-Litt] we find the first proof that $C(\mathbf{B})<\infty$. More recently, one can learn that $C(\mathbf{B}) \leq 7$ from $[\mathbf{A x}]$ (see Theorem 1.21). For the convenience of the reader, however, we include a short (perhaps new) proof that $C(\mathbf{B})<\infty$ in $\S 3$.

As the result of this paper pertains to the theory of quasiconformal mapping, we briefly review the relevant part of that theory here. We shall say that $\kappa$ is a

Received by the editors June 5, 1987 and, in revised form, October 5, 1987.

1980 Mathematics Subject Classification (1985 Revision). Primary 30C60; Secondary 30 C75.

The first author is supported by grant OGPIN-016 of the Natural Sciences and Engineering Research Council of Canada. The second author is supported in part by a grant from the National Science Foundation. 
dilatation if $\kappa$ is a bounded and $\mathscr{L}^{2}$ measurable function on $D$ and $\|\kappa\|_{\infty}<1$. If $\kappa$ is a dilatation, then there is one and only one homeomorphism $H_{\kappa}: \bar{D} \rightarrow \bar{D}$ such that $\bar{\partial} H_{\kappa}(z)=\kappa(z) \cdot \partial H_{\kappa}(z)$ for $\mathscr{L}^{2}$ almost all $z \in D$, and $H_{\kappa}(w)=w$ for all $w \in$ $\{1, i,-1\}$ : a proof of this fundamental statement appears in [Ah1]. Furthermore, we shall say $\kappa$ is an extremal dilatation if $\kappa$ is a dilatation and $\|\kappa\|_{\infty} \leq\left\|\kappa_{1}\right\|_{\infty}$ whenever $\kappa_{1}$ is a dilatation and $H_{\kappa}(w)=H_{\kappa_{1}}(w)$ for all $w \in \mathbf{C}$ such that $|w|=1$. Thus, the statement that $\kappa$ is an extremal dilatation means that $H_{\kappa}$ (the normalized quasiconformal homeomorphism of $D$ having dilatation $\kappa$ ) has the least maximal dilatation among all homeomorphisms of $D$ having the same boundary values as $H_{\kappa}$.

The fundamental characterization of extremal dilatations is due to Richard Hamilton, Edgar Reich, and Kurt Strebel [Ham, Reich-Strebel]. The version of this characterization cited below contains an important elaboration due to Strebel [Strebel], which provides a separation into two alternatives.

TheOREM (HAMILTON, REICH, STREBEL). Suppose $\kappa$ is a dilatation. Then $\kappa$ is an extremal dilatation if and only if one of the following statements is true:

(1) There exist $f \in \mathbf{B}_{1}$ and $k \in[0,1)$ such that $\kappa(z)=k \operatorname{sgn} \overline{f(z)}$ for $\mathscr{L}^{2}$ almost all $z \in D$.

(2) There is a sequence $\left(f_{n}\right)_{n=1}^{\infty}$, of elements of $\mathbf{B}_{1}$, converging to zero uniformly on compact subsets of $D$, such that

$$
\lim _{n \uparrow \infty}\left|\int_{D} f_{n}(z) \kappa(z) d \mathscr{L}^{2}(z)\right|=\|\kappa\|_{\infty} .
$$

Thus, the problem of checking whether a particular dilatation is extremal reduces to that of checking condition (2) above. Our main result, Theorem 1 below, addresses this question.

THEOREM 1. Suppose $\kappa$ is a bounded, $\mathscr{L}^{2}$ measurable function on $D, \pi / 2<$ $\theta<\pi / 2+\arctan (1 / 2 C(\mathbf{B}))$, and $\operatorname{sgn} \kappa(z) \in S(\theta) \cup\{0\}$ for $\mathscr{L}^{2}$ almost all $z \in D$. Then $\kappa$ is an extremal dilatation if and only if there exist $k \in[0,1)$ and $f \in \mathbf{B}_{\mathbf{1}}$ such that

for $\mathscr{L}^{2}$ almost all $z \in D$.

$$
\kappa(z)=k \cdot \operatorname{sgn} \overline{f(z)}
$$

We also emphasize

COROLlARY 2. Suppose $\kappa$ is an extremal dilatation and $\kappa(z)$ is a real number for $\mathscr{L}^{2}$ almost all $z \in D$. Then there exists $k \in(-1,1)$ such that $\kappa(z)=k$ for $\mathscr{L}^{2}$ almost all $z \in D$.

A complete characterization of extremal dilatations which is more explicit than that of Hamilton, Reich and Strebel is not known, although A. Harrington, M. Ortel, E. Reich, K. Strebel and others have obtained characterizations of the extremals within special classes of dilatations: see, for example, $[\mathbf{O}]$ and the references therein. From this work, it is easy to conjecture that the number $\pi / 2+\arctan (1 / 2 C(\mathbf{B}))$ may be replaced by $\pi$ in Theorem 1 , but a proof of this is not known to us.

By selecting a sequence $\left(f_{n}\right)_{n=1}^{\infty}$ from $\mathbf{B}_{1}$ which converges to zero uniformly on compact subsets of $D$, and by approximating $\operatorname{sgn} f_{n}$ on an appropriate sequence of annuli in $D$, it is possible to construct a dilatation $\kappa$, which satisfies condition (2) 
above, and assumes only countably many values. Consequently the measure of the set of arguments of a dilatation is not pertinent to extremality.

2. Proof of Theorem 1. By the theorem of Hamilton, Reich and Strebel, it suffices to prove the following statement.

LEMMA 1. Suppose $\kappa$ is a bounded, $\mathscr{L}^{2}$ measurable function on $D,\|\kappa\|_{\infty}>0$, $\pi / 2<\theta<\pi / 2+\arctan \left(\frac{1}{2 C(\mathbf{B})}\right)$, and $\operatorname{sgn} \kappa(z) \in S(\theta) \cup\{0\}$ for $\mathscr{L}^{2}$ almost all $z \in D$. Also, suppose $\left(f_{n}\right)_{n=1}^{\infty}$ is a sequence from $\mathbf{B}_{1}$ which converges to zero uniformly on compact subsets of $D$. Then

$$
\underset{n \uparrow \infty}{\limsup }\left|\int_{D} f_{n}(z) \kappa(z) d \mathscr{L}^{2}(z)\right|<\|\kappa\|_{\infty}
$$

PROOF. We may assume $\|\kappa\|_{\infty}=1$ and, for each $n=1,2, \ldots$, that

$$
\int_{D} f_{n}(z) \kappa(z) d \mathscr{L}^{2}(z)>0 \quad \text { and } \quad f_{n}(0)=0 .
$$

Choose $\theta_{1}$ so that

$$
\theta<\theta_{1}<\pi / 2+\arctan (1 / 2 C(\mathbf{B})),
$$

and set

$$
\Omega_{n} \equiv\left\{z \in D:-f_{n}(z) \in S\left(\pi-\theta_{1}\right)\right\} .
$$

Then, if $z \in \Omega_{n}$, we have

$$
\operatorname{Re} f_{n}(z) \kappa(z) \leq\left|f_{n}(z) \kappa(z)\right| \cos \left(\theta_{1}-\theta\right) .
$$

So, for each $n$, we have

$$
\begin{aligned}
\int_{D} f_{n}(z) \kappa(z) d \mathscr{L}^{2}(z) & =\operatorname{Re} \int_{D-\Omega_{n}} f_{n}(z) \kappa(z) d \mathscr{L}^{2}(z)+\operatorname{Re} \int_{\Omega_{n}} f_{n}(z) \kappa(z) d \mathscr{L}^{2}(z) \\
& \leq \int_{D-\Omega_{n}}\left|f_{n}(z)\right| d \mathscr{L}^{2}(z)+\cos \left(\theta_{1}-\theta\right) \int_{\Omega_{n}}\left|f_{n}(z)\right| d \mathscr{L}^{2}(z) .
\end{aligned}
$$

Since $\cos \left(\theta_{1}-\theta\right)<1$, it follows that either

$$
\underset{n \uparrow \infty}{\limsup } \int_{D} f_{n}(z) \kappa(z) d \mathscr{L}^{2}(z)<1
$$

as required, or else

$$
\liminf _{n \uparrow \infty} \int_{\Omega_{n}}\left|f_{n}(z)\right| d \mathscr{L}^{2}(z)=0 .
$$

Assuming the second alternative, we define

$$
\mathscr{G}_{n} \equiv\left\{z \in D: \operatorname{Re} f_{n}(z)<0\right\}-\Omega_{n},
$$


and note (since $f_{n}(0)=0$ for all $n$ ) that

$$
\begin{aligned}
\int_{D}\left|\operatorname{Re} f_{n}(z)\right| d \mathscr{L}^{2}(z)= & 2 \cdot \int_{\left\{z \in D: \operatorname{Re} f_{n}(z)<0\right\}}\left|\operatorname{Re} f_{n}(z)\right| d \mathscr{L}^{2}(z) \\
\leq & 2 \cdot \int_{\mathscr{G}_{n}}\left|\operatorname{Re} f_{n}(z)\right| d \mathscr{L}^{2}(z)+2 \cdot \int_{\Omega_{n}}\left|\operatorname{Re} f_{n}(z)\right| d \mathscr{L}^{2}(z) \\
\leq & 2\left|\tan \left(\theta_{1}-\frac{\pi}{2}\right)\right| \cdot \int_{\mathscr{G}_{n}}\left|\operatorname{Im} f_{n}(z)\right| d \mathscr{L}^{2}(z) \\
& +2 \cdot \int_{\Omega_{n}}\left|\operatorname{Re} f_{n}(z)\right| d \mathscr{L}^{2}(z) \\
\leq & 2 C(\mathbf{B})\left|\tan \left(\theta_{1}-\frac{\pi}{2}\right)\right| \int_{D}\left|\operatorname{Re} f_{n}(z)\right| d \mathscr{L}^{2}(z) \\
& +2 \cdot \int_{\Omega_{n}}\left|\operatorname{Re} f_{n}(z)\right| d \mathscr{L}^{2}(z) .
\end{aligned}
$$

Since $2 C(\mathbf{B})\left|\tan \left(\theta_{1}-\pi / 2\right)\right|<1$ and $\liminf { }_{n \uparrow \infty} \int_{\Omega_{n}}\left|f_{n}(z)\right| d \mathscr{L}^{2}(z)=0$, we conclude that $\lim \inf _{n \uparrow \infty} \int_{D}\left|\operatorname{Re} f_{n}(z)\right| d \mathscr{L}^{2}(z)=0$, and hence

$$
\liminf _{n \uparrow \infty} \int_{D}\left|f_{n}(z)\right| d \mathscr{L}^{2}(z)=0
$$

(since $C(\mathbf{B})<\infty$ ). But this contradicts the assumption that $f_{n} \in \mathbf{B}_{1}$ for all $n$. Therefore, the second alternative is impossible and Theorem 1 follows.

3. A proof that $C(\mathbf{B})<\infty$. Let $f$ be an element of $\mathbf{B}$, and, without loss of generality, assume that $f(0)=0$. Define $u \equiv \operatorname{Re} f$, and $r_{n} \equiv 1-2^{-n}$ for $n=1,2, \ldots$ If $|z|<r_{n+1}$, then

$$
f(z)=(2 \pi)^{-1} \int_{0}^{2 \pi} \frac{r_{n+1} e^{i t}+z}{r_{n+1} e^{i t}-z} u\left(r_{n+1} e^{i t}\right) d \mathscr{L}^{1}(t)
$$

and easy estimates show that, for $n=1,2, \ldots$,

$$
\int_{0}^{2 \pi}\left|f\left(r_{n} e^{i \theta}\right)-f\left(r_{n-1} e^{i \theta}\right)\right| d \mathscr{L}^{1}(\theta) \leq 2^{3 / 2} \int_{0}^{2 \pi}\left|u\left(r_{n+1} e^{i \theta}\right)\right| d \mathscr{L}^{1}(\theta)
$$

Moreover, by using the monotonicity of integral means to estimate the integrals over annuli, we find

$$
\frac{1}{5} \sum_{n=1}^{\infty} 2^{-n} \int_{0}^{2 \pi}\left|f\left(r_{n+1} e^{i \theta}\right)\right| d \mathscr{L}^{1}(\theta) \leq\|f\|_{1} \leq \sum_{N=1}^{\infty} 2^{-n} \int_{0}^{2 \pi}\left|f\left(r_{n} e^{i \theta}\right)\right| d \mathscr{L}^{1}(\theta)
$$


together with the analogous inequalities for $u$. Since $f(0)=0$, it follows that

$$
\begin{aligned}
\|f\|_{1} & \leq \sum_{n=1}^{\infty} 2^{-n} \int_{0}^{2 \pi}\left|f\left(r_{n} e^{i \theta}\right)\right| d \mathscr{L}^{1}(\theta) \\
& \leq \sum_{n=1}^{\infty} 2^{-n} \sum_{k=1}^{n} \int_{0}^{2 \pi}\left|f\left(r_{k} e^{i \theta}\right)-f\left(r_{k-1} e^{i \theta}\right)\right| d \mathscr{L}^{1}(\theta) \\
& =2 \sum_{k=1}^{\infty} 2^{-k} \int_{0}^{2 \pi}\left|f\left(r_{k} e^{i \theta}\right)-f\left(r_{k-1} e^{i \theta}\right)\right| d \mathscr{L}^{1}(\theta) \\
& \leq 2(2)^{3 / 2} \sum_{k=1}^{\infty} 2^{-k} \int_{0}^{2 \pi}\left|u\left(r_{k+1} e^{i \theta}\right)\right| d \mathscr{L}^{1}(\theta) \\
& \leq 10(2)^{3 / 2} \int_{D}|u(z)| d \mathscr{L}^{2}(z)
\end{aligned}
$$

\section{REFERENCES}

[Ahl] L. V. Ahlfors, Lectures on quasiconformal mappings, Van Nostrand, 1966.

[Ax] S. Axler, Bergmun spaces and their operators (to appear).

[Ham] R. S. Hamilton, Extremal quasiconformal mappings with prescribed boundary values, Trans. Amer. Math. Soc. 138 (1969), 399-406.

[Har-Litt] G. H. Hardy and J. E. Littlewood, Some properties of conjugate functions, J. Reine Angew. Math. 167 (1931), 405-423.

[O] M. Ortel, Extremal quasiconformal mappings with angular complex dilatation, Indiana Univ. Math. J. 31 (1982), 435-447.

[Reich-Strebel] E. Reich and K. Strebel, Extremal quasiconformal mappings with given boundary values, Contributions to Analysis, a collection of papers dedicated to Lipman Bers, pp. 375391, Academic Press, New York, 1974.

[Strebel] K. Strebel, On quadratic differentials and extremal quasiconformal mappings, Proc. Internat. Congress Math., Vancouver, 1974, vol. 2, Canad. Math. Congr., 1975, pp. 223-227.

Department of Mathematics, University of HaWail, Honolulu, haWail 96822 